\title{
Large Poisson-Voronoi Cells and Crofton Cells
}

\author{
Daniel Hug* \\ Matthias Reitzner ${ }^{\dagger}$ \\ Rolf Schneider
}

\begin{abstract}
It is proved that the shape of the typical cell of a stationary Poisson-Voronoi tessellation in Euclidean space, under the condition that the volume of the typical cell is large, must be close to spherical shape, with high probability. The same holds if the volume is replaced by the surface area or other suitable functionals. Similar results hold for the zero cell of a stationary and isotropic Poisson hyperplane tessellation.
\end{abstract}

\section{Introduction}

In this paper, we continue a line of research that began with a problem of D.G. Kendall (see the foreword to the first edition of [17]). He conjectured that the shape of the zero cell (or Crofton cell) of a stationary and isotropic Poisson line process in the plane, given that the area of the cell tends to infinity, must tend to circular shape. Contributions to Kendall's question are due to Miles [11] and Goldman [3], and the conjecture was proved by Kovalenko [8], [10]. In [7], Kovalenko's result was extended to higher dimensions and to stationary, but not necessarily isotropic Poisson hyperplane processes. It was also strengthened, by estimating the probability of large deviations from spherical shape, given that the volume of the zero cell lies in a prescribed interval. In the present paper, we prove an analogous result for the typical cell of a stationary Poisson-Voronoi tessellation of $d$-dimensional space. This extends and strengthens a result of Kovalenko [9] in the planar case. We further generalize this result by considering, in addition to the volume functional, also the $k$ th intrinsic volume, $k=1, \ldots, d-1$. This includes cells of large surface area or of large mean width. The result from [7] on Crofton cells of stationary Poisson hyperplane processes with large volume is also extended to the $k$ th intrinsic volume, but only for $k \geq 2$ and under the additional assumption of isotropy. For both types of random polytopes, Poisson-Voronoi cells and isotropic Crofton cells, we can also replace (somewhat easier) the condition of large volume by the condition of large inradius. This is suggested by considerations of Miles [11] on Crofton cells in the plane and by the work of Calka [1] on planar Poisson-Voronoi tessellations. Finally, we mention here that cells of large volume in Poisson-Delaunay tessellations were treated in [6]; such cells tend to be regular simplices.

Let $A$ be a locally finite point set in Euclidean space $\mathbb{R}^{d}$ (with scalar product $\langle\cdot, \cdot\rangle$ and norm $\|\cdot\|)$, where $d \geq 2$. For $\boldsymbol{x} \in A$, the Voronoi cell of $\boldsymbol{x}$ with respect to $A$ is defined by

$$
C(\boldsymbol{x}, A):=\left\{\boldsymbol{y} \in \mathbb{R}^{d}:\|\boldsymbol{y}-\boldsymbol{x}\| \leq\|\boldsymbol{y}-\boldsymbol{a}\| \text { for all } \boldsymbol{a} \in A\right\} .
$$

${ }^{*}$ Postal address of the authors: Mathematisches Institut, Albert-Ludwigs-Universität, Eckerstr. 1, D-79104 Freiburg, Germany. Email addresses: \{daniel.hug, matthias.reitzner, rolf.schneider\} @math.uni-freiburg.de

${ }^{\dagger}$ Postal address also: Institut für Analysis und Technische Mathematik, Technische Universität Wien, Wiedner Hauptstrasse 8 - 10, A-1040 Wien, Austria. Email address: mreitzne@mail.zserv.tuwien.ac.at 
Let $\tilde{X}$ be a stationary Poisson point process of intensity $\lambda>0$ in $\mathbb{R}^{d}$. (In treating simple point processes, we conveniently identify a simple counting measure with its support.) Then

$$
X:=\{C(\boldsymbol{x}, \tilde{X}): \boldsymbol{x} \in \tilde{X}\}
$$

is the Poisson-Voronoi tessellation derived from $\tilde{X}$. Let $Z$ denote the typical cell of $X$ (we recall its definition in Section 2).

For a convex body (non-empty, compact, convex set) $K \subset \mathbb{R}^{d}$, we denote the volume by $v_{d}(K)$. The (conveniently renormalized) intrinsic volumes $v_{0}(K), \ldots, v_{d-1}(K)$ can be defined by means of the Steiner formula

$$
v_{d}\left(K+\epsilon B^{d}\right)=\sum_{k=0}^{d} \epsilon^{d-k}\left(\begin{array}{l}
d \\
k
\end{array}\right) v_{k}(K), \quad \epsilon \geq 0 .
$$

Here $B^{d}:=\left\{\boldsymbol{x} \in \mathbb{R}^{d}:\|\boldsymbol{x}\| \leq 1\right\}$ is the unit ball. Equivalently, $v_{i}(K)$ is the mixed volume $V\left(K[i], B^{d}[d-i]\right)$ of $i$ copies of $K$ and $d-i$ copies of $B^{d}$. The functional $W_{j}=v_{d-j}$ is known as the $j$ th quermassintegral. In particular, $d v_{d-1}$ is the surface area, and $\left(2 / \kappa_{d}\right) v_{1}$ is the mean width; here $\kappa_{d}:=v_{d}\left(B^{d}\right)$. More information is found in [15].

Let $K \subset \mathbb{R}^{d}$ be a compact set with $\boldsymbol{o} \in K$ and containing more than one point. In order to measure the deviation of $K$ from a ball with centre $\boldsymbol{o}$, we define

$$
\delta(K):=\frac{R_{o}-\rho_{o}}{R_{o}+\rho_{o}}
$$

where $R_{o}$ is the radius of the smallest ball with centre $\boldsymbol{o}$ containing $K$ and $\rho_{o}$ is the radius (possibly zero) of the largest ball with centre $\boldsymbol{o}$ contained in $K$.

By $\mathrm{P}$ we denote the underlying probability, and $\mathrm{P}(\cdot \mid \cdot)$ is a conditional probability.

Theorem 1. Let $X$ denote the Poisson-Voronoi tessellation derived from a stationary Poisson point process with intensity $\lambda>0$ in $\mathbb{R}^{d}$; let $Z$ be its typical cell. Let $k \in\{1, \ldots, d\}$. There is a positive constant $c_{0}$ depending only on the dimension $d$ such that the following is true. If $\epsilon \in(0,1)$ and $I=[a, b)$ is any interval (possibly $b=\infty$ ) with $a^{d / k} \lambda \geq \sigma_{0}>0$, then

$$
\mathrm{P}\left(\delta(Z) \geq \epsilon \mid v_{k}(Z) \in I\right) \leq c \exp \left\{-c_{0} \epsilon^{(d+3) / 2} a^{d / k} \lambda\right\}
$$

where $c$ is a constant depending only on $d, \epsilon$ and $\sigma_{0}$.

In particular,

$$
\lim _{a \rightarrow \infty} \mathrm{P}\left(\delta(Z) \geq \epsilon \mid v_{k}(Z) \geq a\right)=0
$$

for every $\epsilon>0$, but Theorem 1 provides much stronger information.

Theorem 1 will be proved in Section 6, after preliminary explanations in Section 2 and preparations in Sections 3 to 5.

In [7], a similar result was obtained for the volume of the zero cell (also called Crofton cell) of the tessellation generated by a stationary Poisson hyperplane process. We will indicate in Section 7 how, under the additional assumption of isotropy, this result can be extended to the $k$ th intrinsic volume, $k=2, \ldots, d$. As in [7], we measure the deviation of the shape of a 
convex body $K \subset \mathbb{R}^{d}$ with interior points from spherical shape by $r_{B^{d}}$, which we abbreviate by $r_{d}$, thus

$$
r_{d}(K):=\min \left\{s / r-1: r B^{d}+\boldsymbol{z} \subset K \subset s B^{d}+\boldsymbol{z}, \boldsymbol{z} \in \mathbb{R}^{d}, r, s>0\right\} .
$$

Theorem 2. Let $Z_{o}$ be the zero cell of the tessellation induced by a stationary isotropic Poisson hyperplane process in $\mathbb{R}^{d}$ with intensity $\lambda>0$. Let $k \in\{2, \ldots, d\}$. There is a positive constant $c_{0}$ depending only on the dimension $d$ such that the following is true. If $\epsilon \in(0,1)$ and $I=[a, b)$ is any interval (possibly $b=\infty$ ) with $a^{1 / k} \lambda \geq \sigma_{0}>0$, then

$$
\mathrm{P}\left(r_{d}\left(Z_{o}\right) \geq \epsilon \mid v_{k}\left(Z_{o}\right) \in I\right) \leq c \exp \left\{-c_{0} \epsilon^{(d+3) / 2} a^{1 / k} \lambda\right\}
$$

where $c$ is a constant depending only on $d, \epsilon$ and $\sigma_{0}$.

The case of the volume is included here for $k=d$. We remark that in this case the inequality of Theorem 2 is sharper (in its dependence on $\epsilon$ ) than Theorem 1 of [7], specialized to the isotropic case. The reason for this improvement lies in the fact that in the isotropic case sharper stability estimates from convex geometry are available. Unfortunately, our method of proof does not permit us to treat the case $k=1$, which in the plane is the case of the perimeter, already studied by Miles [11] in his heuristic approach.

In addition to $\rho_{o}(K)$ defined above, we denote by $\rho(K)$ the radius of the largest ball contained in the convex body $K$.

Theorem 3. Let $Z$ and $Z_{o}$ be defined as in Theorems 1 and 2, respectively. There is a positive constant $c_{0}$ depending only on the dimension $d$ such that the following is true. If $\epsilon \in(0,1)$ and $I=[a, b)$ is any interval (possibly $b=\infty$ ) with $a^{d} \lambda \geq \sigma_{0}>0$ in the case of $Z$, respectively $a \lambda \geq \sigma_{0}>0$ in the case of $Z_{o}$, then

$$
\mathrm{P}\left(\delta(Z) \geq \epsilon \mid \rho_{o}(Z) \in I\right) \leq c \exp \left\{-c_{0} \epsilon^{(d+1) / 2} a^{d} \lambda\right\}
$$

and

$$
\mathrm{P}\left(r_{d}\left(Z_{o}\right) \geq \epsilon \mid \rho\left(Z_{o}\right) \in I\right) \leq c \exp \left\{-c_{0} \epsilon^{(d+1) / 2} a \lambda\right\}
$$

where $c$ is a constant depending only on $d, \epsilon$ and $\sigma_{0}$.

The proof will be sketched in Section 8 .

\section{The typical cell of a Poisson-Voronoi tessellation}

We recall the definition of the typical cell of the Poisson-Voronoi mosaic $X$, generated by the stationary Poisson point process $\tilde{X}$. In general, the notion of the typical cell of a stationary random mosaic requires the choice of a centroid function (e.g., see Møller [12, Section 3.2]), but for Voronoi cells there is a canonical choice, the nucleus. Let $\mathcal{K}_{o}^{d}$ denote the space of convex bodies $K$ in $\mathbb{R}^{d}$ with $\boldsymbol{o} \in K$, equipped with the Hausdorff metric and corresponding Borel structure. The distribution $\mathbb{Q}$ of the typical cell of $X$ can be defined by

$$
\mathbb{Q}(\mathcal{A})=\frac{1}{\lambda} \mathrm{E} \sum_{\boldsymbol{x} \in \tilde{X}} \mathbf{1}_{\mathcal{A}}(C(\boldsymbol{x}, \tilde{X})-\boldsymbol{x}) \mathbf{1}_{B}(\boldsymbol{x})
$$


(E denotes mathematical expectation) for Borel sets $\mathcal{A} \subset \mathcal{K}_{o}^{d}$, where $B \subset \mathbb{R}^{d}$ is an arbitrary Borel set with $\lambda_{d}(B)=1$; here $\lambda_{d}$ denotes Lebesgue measure in $\mathbb{R}^{d}$. The distribution $\mathbb{Q}$ is commonly interpreted as the conditional distribution of $C(\boldsymbol{o}, \tilde{X})$ given that $\boldsymbol{o} \in \tilde{X}$. An intuitive interpretation follows from the fact that stationary Poisson-Voronoi tessellations are mixing ([16, Satz 6.4.1]) and hence ergodic. This implies that

$$
\mathbb{Q}(\mathcal{A})=\lim _{r \rightarrow \infty} \frac{\operatorname{card}\left\{\boldsymbol{x} \in \tilde{X} \cap r B^{d}: C(\boldsymbol{x}, \tilde{X})-\boldsymbol{x} \in \mathcal{A}\right\}}{\operatorname{card}\left(\tilde{X} \cap r B^{d}\right)}
$$

holds with probability one.

Since $\tilde{X}$ is a stationary Poisson process, it follows from Slivnyak's theorem that the typical cell of the Poisson-Voronoi mosaic $X$ is stochastically equivalent to the random polytope

$$
Z=C(\boldsymbol{o}, \tilde{X} \cup\{\boldsymbol{o}\})
$$

(see [12, Remark 4.1.1]; see also [13]). Hence, we can consider $Z$ as the typical cell of $X$, and for this we obtain a convenient representation. For $\boldsymbol{x} \in \mathbb{R}^{d}$, we define

$$
\begin{aligned}
H(\boldsymbol{x}) & :=\left\{\boldsymbol{y} \in \mathbb{R}^{d}:\langle\boldsymbol{y}, \boldsymbol{x}\rangle=\|\boldsymbol{x}\|^{2} / 2\right\}, \\
H^{-}(\boldsymbol{x}) & :=\left\{\boldsymbol{y} \in \mathbb{R}^{d}:\langle\boldsymbol{y}, \boldsymbol{x}\rangle \leq\|\boldsymbol{x}\|^{2} / 2\right\},
\end{aligned}
$$

so that $H(\boldsymbol{x})$ is the mid hyperplane of $\boldsymbol{o}$ and $\boldsymbol{x}$. Then

$$
Z=\bigcap_{\boldsymbol{x} \in \tilde{X}} H^{-}(\boldsymbol{x})
$$

thus $Z$ is the zero cell of the tessellation induced by the Poisson hyperplane process

$$
Y:=\{H(\boldsymbol{x}): \boldsymbol{x} \in \tilde{X}\} .
$$

The intensity measure $\mathrm{E} Y(\cdot)$ of this process can be represented as follows. For a Borel set $\mathcal{A}$ in the space of hyperplanes, we have

$$
\begin{aligned}
\operatorname{E} Y(\mathcal{A}) & =\operatorname{Ecard}(\mathcal{A} \cap Y)=\operatorname{Ecard}\{\boldsymbol{x} \in \tilde{X}: H(\boldsymbol{x}) \in \mathcal{A}\} \\
& =\lambda \cdot \lambda_{d}\left(\left\{\boldsymbol{x} \in \mathbb{R}^{d}: H(\boldsymbol{x}) \in \mathcal{A}\right\}\right) .
\end{aligned}
$$

Writing

$$
H(\boldsymbol{u}, t):=\left\{\boldsymbol{y} \in \mathbb{R}^{d}:\langle\boldsymbol{y}, \boldsymbol{u}\rangle=t\right\}, \quad H^{-}(\boldsymbol{u}, t):=\left\{\boldsymbol{y} \in \mathbb{R}^{d}:\langle\boldsymbol{y}, \boldsymbol{u}\rangle \leq t\right\}
$$

for $\boldsymbol{u} \in S^{d-1}:=\left\{\boldsymbol{x} \in \mathbb{R}^{d}:\|\boldsymbol{x}\|=1\right\}$ and $t \in \mathbb{R}$, and introducing polar coordinates, we get

$$
\mathrm{E} Y(\cdot)=2^{d} \lambda \int_{S^{d-1}} \int_{0}^{\infty} \mathbf{1}\{H(\boldsymbol{u}, t) \in \cdot\} t^{d-1} \mathrm{~d} t \sigma(\mathrm{d} \boldsymbol{u})
$$

where $\sigma$ denotes spherical Lebesgue measure on the unit sphere $S^{d-1}$.

In particular, for $K \in \mathcal{K}_{o}^{d}$ let $\mathcal{H}_{K}$ be the set of all hyperplanes $H \subset \mathbb{R}^{d}$ with $H \cap K \neq \emptyset$. Then (1) gives

$$
\operatorname{E} Y\left(\mathcal{H}_{K}\right)=2^{d} \lambda U(K)
$$

with

$$
U(K):=\frac{1}{d} \int_{S^{d-1}} h(K, \boldsymbol{u})^{d} \sigma(\mathrm{d} \boldsymbol{u})
$$


where $h(K, \cdot)$ is the support function of $K$. Writing

$$
\Phi(K):=\left\{\boldsymbol{y} \in \mathbb{R}^{d}: H(2 \boldsymbol{y}) \cap K \neq \emptyset\right\},
$$

we have $U(K)=\lambda_{d}(\Phi(K))$. The star body $\Phi(K)$ is the union of all closed balls having a diameter segment $[\boldsymbol{o}, \boldsymbol{x}]$ with $\boldsymbol{x} \in K$.

The relation (2), together with the Poisson property of the hyperplane process $Y$, implies that

$$
\mathrm{P}\left(Y\left(\mathcal{H}_{K}\right)=k\right)=\frac{\left[2^{d} U(K) \lambda\right]^{k}}{k !} \exp \left\{-2^{d} U(K) \lambda\right\}
$$

for $K \in \mathcal{K}_{o}^{d}$ and $k \in \mathbb{N}_{0}$.

It is now clear that we are in a similar situation as in [7]. There, the zero cell of a stationary (not necessarily isotropic) Poisson hyperplane process, with intensity measure given by [7, (2)], was studied. This process is now replaced by the isotropic, non-stationary hyperplane process $Y$, with intensity measure given by (1). The functional $U(K)$, defined by (3), will play the role of the mixed volume $V_{1}(B, K)$ in [7] (up to dimensional factors). In addition to the volume functional considered in [7], we now treat general intrinsic volumes. All these differences require a number of changes and new arguments, but also some parallel reasoning is possible. In the latter cases, we will be brief and just list how the arguments of [7] have to be modified.

\section{$3 \quad$ A stability estimate}

For $K \in \mathcal{K}_{o}^{d}$, we trivially have $K \subset \Phi(K)$, hence $U(K) \geq v_{d}(K)$. Here equality holds if and only if $K$ is a ball with centre $\boldsymbol{o}$ (this follows from the considerations below, but can also be shown directly). A similar inequality can be obtained for the other intrinsic volumes. In the following, we write $h(K, \cdot)=: h_{K}$ for the support function of $K$. Integrations with respect to $\sigma$ extend over $S^{d-1}$. By Hölder's inequality,

$$
U(K) \geq \frac{1}{d}\left(d \kappa_{d}\right)^{1-d}\left(\int h_{K} \mathrm{~d} \sigma\right)^{d}
$$

and since

$$
\int h_{K} \mathrm{~d} \sigma=d v_{1}(K)
$$

we get

$$
U(K)^{1 / d} \geq \kappa_{d}^{(1-d) / d} v_{1}(K)
$$

A well-known inequality (e.g., [15, p. 334]) says that

$$
v_{1}(K)^{k} \geq \kappa_{d}^{k-1} v_{k}(K)
$$

for $k=1, \ldots, d$. Hence,

$$
U(K) \geq \kappa_{d}^{1-d / k} v_{k}(K)^{d / k} .
$$

Equality for a number $k \in\{1, \ldots, d\}$ holds if and only if $K$ is a ball with centre $\boldsymbol{o}$. We will need an improved version of (6), in the form of a stability estimate.

Lemma 1. For $K \in \mathcal{K}_{o}^{d}$ and $k \in\{1, \ldots, d\}$,

$$
U(K) \geq\left(1+\gamma \delta(K)^{(d+3) / 2}\right) \kappa_{d}^{1-d / k} v_{k}(K)^{d / k},
$$


where $\gamma$ is a positive constant depending only on the dimension $d$.

Proof. We assume that $K$ contains more than one point; otherwise the assertion is trivial. In order to improve Hölder's inequality, we use Lemma 4.2 of Gardner and Vassallo [2]. There we put $m=2, f_{0}=f_{2}=1, f_{1}=h_{K}, w_{1}=1 / d, w_{2}=(d-1) / d$ (hence $w=1 / d$ ), and obtain

$$
\begin{aligned}
& 1-\frac{\int h_{K} \mathrm{~d} \sigma}{\left(\int h_{K}^{d} \mathrm{~d} \sigma\right)^{1 / d}\left(\int 1 \mathrm{~d} \sigma\right)^{(d-1) / d}} \\
& \geq \frac{1}{d} \int\left[\frac{h_{K}^{d / 2}}{\left(\int h_{K}^{d} \mathrm{~d} \sigma\right)^{1 / 2}}-\frac{1}{\left(\int 1 \mathrm{~d} \sigma\right)^{1 / 2}}\right]^{2} \mathrm{~d} \sigma=: \beta(K) .
\end{aligned}
$$

Using $(1-\beta)(1+\beta) \leq 1$, we deduce that

$$
U(K)^{1 / d} \geq(1+\beta(K)) \kappa_{d}^{(1-d) / d} v_{1}(K) .
$$

Next, we establish an estimate of the form $\beta(K) \geq c \delta(K)^{\alpha}$ with $\alpha>0$. For this, we argue similarly as in the proof of Lemma 1 in [5] (which is reproduced in [4], see inequality (2.3.3)).

From now on in this paper, $c_{1}, c_{2}, \ldots$ denote constants depending only on the dimension, except in those cases where other dependences are explicitly indicated.

Let $K \in \mathcal{K}_{o}^{d}$ be given. Since $\beta$ and $\delta$ are invariant under dilatations, we can assume that

$$
\int h_{K}^{d} \mathrm{~d} \sigma=\int 1 \mathrm{~d} \sigma=d \kappa_{d}
$$

In this case, by (6),

$$
\kappa_{d}=U(K) \geq \kappa_{d}^{1-d} v_{1}(K)^{d} \geq c_{1} D(K)^{d},
$$

where $D$ denotes the diameter, hence $D(K) \leq c_{2}$ and therefore

$$
h_{K} \leq c_{2} .
$$

Moreover,

$$
\beta(K)=c_{3} \int\left(h_{K}^{d / 2}-1\right)^{2} \mathrm{~d} \sigma .
$$

Suppose that $\rho_{o} B^{d} \subset K \subset R_{o} B^{d}$, where $\rho_{o}$ is maximal and $R_{o}$ is minimal. It follows from (9) that $\rho_{o} \leq 1 \leq R_{o}$.

Case 1: $R_{o}-1 \geq 1-\rho_{o}$.

We put $R_{o}=1+h$, then

$$
\delta(K)=\frac{R_{o}-\rho_{o}}{R_{o}+\rho_{o}} \leq 2 h .
$$

There exists a vector $\boldsymbol{u}_{0} \in S^{d-1}$ such that $h_{K}\left(\boldsymbol{u}_{0}\right)=R_{o}=1+h$, and the point $\boldsymbol{p}=(1+h) \boldsymbol{u}_{0}$ belongs to $K$. For $\boldsymbol{u} \in S^{d-1}$, let $E(\boldsymbol{u})$ be the hyperplane through $\boldsymbol{p}$ and orthogonal to $\boldsymbol{u}$. Let $\omega$ denote the angle between $\boldsymbol{u}$ and $\boldsymbol{u}_{0}$, and let $\omega_{0}$ be the angle between $\boldsymbol{u}_{0}$ and any $\boldsymbol{v}$ such that $E(\boldsymbol{v})$ is tangent to $B^{d}$. Then $\cos \omega_{0}=1 /(1+h)$ and (with $\sigma_{d-1}:=(d-1) \kappa_{d-1}$ )

$$
\int\left(h_{K}^{d / 2}-1\right)^{2} \mathrm{~d} \sigma \geq \sigma_{d-1} \int_{0}^{\omega_{0}}\left\{[(1+h) \cos \omega]^{d / 2}-1\right\}^{2}(\sin \omega)^{d-2} \mathrm{~d} \omega .
$$


We set $(1+h)^{d / 2}-1=: a$ and substitute $[(1+h) \cos \omega]^{d / 2}-1=a x$, to obtain

$$
\begin{aligned}
& \int\left(h_{K}^{d / 2}-1\right)^{2} \mathrm{~d} \sigma \\
& \geq \frac{2 \sigma_{d-1} a^{3}}{d(1+h)^{d-2}} \int_{0}^{1} x^{2}\left[(1+h)^{2}-(a x+1)^{4 / d}\right]^{(d-3) / 2}(a x+1)^{(2-d) / d} \mathrm{~d} x .
\end{aligned}
$$

By (10), we can estimate

$$
(1+h)^{2-d} \geq c_{4}
$$

and, for $0 \leq x \leq 1$,

$$
(a x+1)^{(2-d) / d} \geq(a+1)^{(2-d) / d}=(1+h)^{(2-d) / 2} \geq c_{4}^{1 / 2} .
$$

This gives

$$
\beta(K) \geq c_{5} a^{3} \int_{0}^{1} x^{2}\left[(1+h)^{2}-(a x+1)^{4 / d}\right]^{(d-3) / 2} \mathrm{~d} x
$$

The function

$$
f(x):=(1+h)^{2}-(a x+1)^{4 / d}, \quad 0 \leq x \leq 1,
$$

satisfies $f(0)=h(h+2), f(1)=0$, and $f^{\prime}(1)=-(4 / d) a(1+h)^{(4-d) / 2}$. It is convex for $d \geq 4$ and concave for $d=2,3$. For $d \geq 4$ we deduce that

$$
f(x) \geq \frac{4}{d} a(1+h)^{(4-d) / 2}(1-x) \geq c_{6} a(1-x),
$$

and for $d=2,3$ we get

$$
f(x) \geq h(h+2)(1-x) \geq h(1-x) .
$$

Together with

$$
a=(1+h)^{d / 2}-1 \geq \frac{d}{2} h
$$

this yields

$$
\beta(K) \geq c_{7} h^{(d+3) / 2} .
$$

From (11) we now get

$$
\beta(K) \geq c_{8} \delta(K)^{(d+3) / 2} .
$$

Case 2: $R_{o}-1<1-\rho_{o}$.

We put $\rho_{o}=1-h$, then $R_{o}<1+h$, hence

$$
K \subset(1+h) B^{d}
$$

and

$$
\delta(K) \leq 2 h .
$$

There is a vector $\boldsymbol{u}_{0} \in S^{d-1}$ such that $h_{K}\left(\boldsymbol{u}_{0}\right)=\rho_{o}=1-h$, and the hyperplane $G$ through $\rho_{o} \boldsymbol{u}_{0}$ orthogonal to $\boldsymbol{u}_{0}$ supports $K$. Let $\boldsymbol{p} \in\left(\partial(1+h) B^{d}\right) \cap G$. Let $\boldsymbol{u}_{1} \in S^{d-1}$ be the vector, positively spanned by $\boldsymbol{u}_{0}$ and $\boldsymbol{p}$, that is orthogonal to a support plane of $B^{d}$ through $\boldsymbol{p}$. Let 
$\omega_{0}$ be the angle between $\boldsymbol{u}_{0}$ and $\boldsymbol{u}_{1}$. For $\boldsymbol{u} \in S^{d-1}$, let $\omega_{\boldsymbol{u}}$ be the angle between $\boldsymbol{u}_{0}$ and $\boldsymbol{u}$. If $\omega_{\boldsymbol{u}} \leq \omega_{0}$, then $h_{K}(\boldsymbol{u}) \leq 1$, hence $1-h_{K}(\boldsymbol{u})^{d / 2} \geq 1-h_{K}(\boldsymbol{u}) \geq 0$ and thus

$$
\beta(K) \geq c_{3} \int\left(1-h_{K}(\boldsymbol{u})\right)^{2} \mathbf{1}\left\{\omega_{\boldsymbol{u}} \leq \omega_{0}\right\} \sigma(\mathrm{d} \boldsymbol{u}) .
$$

Now exactly the argument of Case 2 in [5, pp. 71-72] leads to

$$
\beta(K) \geq c_{9} h^{(d+3) / 2},
$$

which together with (13) gives

$$
\beta(K) \geq c_{10} \delta(K)^{(d+3) / 2}
$$

In each case, we conclude from (8) and (5) the inequality (7).

\section{Probabilities involving small intervals}

It is easy to obtain a lower bound for the probability of the event $v_{k}(Z) \geq a$, where $a \geq 0$. In fact, let $B_{a}:=\left(a / \kappa_{d}\right)^{1 / k} B^{d}$, then $v_{k}\left(B_{a}\right)=a$ and hence

$$
\mathrm{P}\left(v_{k}(Z) \geq a\right) \geq \mathrm{P}\left(Y\left(\mathcal{H}_{B_{a}}\right)=0\right)=\exp \left\{-2^{d} U\left(B_{a}\right) \lambda\right\}=\exp \left\{-2^{d} \kappa_{d}^{1-d / k} a^{d / k} \lambda\right\} .
$$

In this section we prove an inequality which replaces the previous one if $v_{k}(Z)$ is contained in a small interval.

For $A \subset \mathbb{R}^{d}$ we define

$$
Z(A):=\bigcap_{\boldsymbol{x} \in A} H^{-}(\boldsymbol{x})
$$

and set $Z\left(\boldsymbol{x}_{1}, \ldots, \boldsymbol{x}_{n}\right):=Z\left(\left\{\boldsymbol{x}_{1}, \ldots, \boldsymbol{x}_{n}\right\}\right)$. For the random polytope $Z(\tilde{X})$, the typical cell of $X$, we retain the notation $Z$.

Let $k \in\{1, \ldots, d\}$ be fixed.

Lemma 2. For each $\beta>0$, there are constants $h_{0}>0, N \in \mathbb{N}$ and $c_{11}>0$, depending only on $\beta$ and $d$, such that for $a>0$ and $0<h<h_{0}$,

$$
\mathrm{P}\left(v_{k}(Z) \in a(1,1+h)\right) \geq c_{11} h\left(a^{d / k} \lambda\right)^{N} \exp \left\{-(1+\beta)^{d} 2^{d} \kappa_{d}^{1-d / k} a^{d / k} \lambda\right\} .
$$

Proof. Let $\beta>0$ and $a>0$ be given. For $n \geq 2$ we define

$$
\begin{aligned}
\mathcal{Q}_{n}:= & \left\{\left(\boldsymbol{x}_{1}, \ldots, \boldsymbol{x}_{n-1}, \boldsymbol{u}\right) \in\left((1+\beta / 2) B^{d}\right)^{n-1} \times S^{d-1}:\right. \\
& \left.Z\left(\boldsymbol{x}_{1}, \ldots, \boldsymbol{x}_{n-1}, \boldsymbol{u}\right) \subset 2^{-1}(1+\beta / 2) B^{d}, v_{k}\left(Z\left(\boldsymbol{x}_{1}, \ldots, \boldsymbol{x}_{n-1}, \boldsymbol{u}\right)\right) \geq 2^{-k} \kappa_{d}\right\} .
\end{aligned}
$$

A continuity argument shows that we can choose $N=N(\beta, d) \in \mathbb{N}$ (sufficiently large) so that

$$
\int_{S^{d-1}} \int_{\mathbb{R}^{d}} \ldots \int_{\mathbb{R}^{d}} \mathbf{1}\left\{\left(\boldsymbol{x}_{1}, \ldots, \boldsymbol{x}_{N-1}, \boldsymbol{u}\right) \in \mathcal{Q}_{N}\right\} \mathrm{d} \boldsymbol{x}_{1} \ldots \mathrm{d} \boldsymbol{x}_{N-1} \sigma(\mathrm{d} \boldsymbol{u})=: c_{12}(d, \beta)>0 .
$$


If $\left(\boldsymbol{x}_{1}, \ldots, \boldsymbol{x}_{N-1}, \boldsymbol{u}\right) \in \mathcal{Q}_{N}$, then

$$
v_{k}\left(Z\left(\boldsymbol{x}_{1}, \ldots, \boldsymbol{x}_{N-1}, \boldsymbol{u}\right)\right) \leq(1+\beta / 2)^{k} 2^{-k} \kappa_{d} .
$$

Define $h_{0}=h_{0}(\beta)>0$ by $\left(1+h_{0}\right)^{1 / k}(1+\beta / 2)=1+\beta$ and suppose that $0<h<h_{0}$. Putting $\left(a / \kappa_{d}\right)^{1 / k} B^{d}=B_{a}$ as before, we can estimate (recall that $\tilde{X}(\omega)$, a realization of $\tilde{X}$, denotes a simple counting measure and also its support)

$$
\begin{aligned}
& \mathrm{P}\left(v_{k}(Z) \in a(1,1+h)\right) \\
\geq & \mathrm{P}\left(\tilde{X}\left(2(1+\beta) B_{a}\right)=N, Z\left(\tilde{X} \cap 2(1+\beta) B_{a}\right) \subset(1+\beta) B_{a},\right. \\
& \left.v_{k}\left(Z\left(\tilde{X} \cap 2(1+\beta) B_{a}\right)\right) \in a(1,1+h)\right) \\
= & \mathrm{P}\left(\tilde{X}\left(2(1+\beta) B_{a}\right)=N\right) \mathrm{P}\left(Z\left(\tilde{X} \cap 2(1+\beta) B_{a}\right) \subset(1+\beta) B_{a},\right. \\
& \left.v_{k}\left(Z\left(\tilde{X} \cap 2(1+\beta) B_{a}\right)\right) \in a(1,1+h) \mid \tilde{X}\left(2(1+\beta) B_{a}\right)=N\right) .
\end{aligned}
$$

Since $\tilde{X}$ is a stationary Poisson process with intensity $\lambda$, we obtain (using Satz 3.2.3(b) of [16])

$$
\begin{aligned}
& \mathrm{P}\left(v_{k}(Z) \in a(1,1+h)\right) \\
& \geq \frac{\lambda^{N}}{N !} \exp \left\{-v_{d}\left(2(1+\beta) B_{a}\right) \lambda\right\} \\
& \quad \times \int_{\mathbb{R}^{d}} \ldots \int_{\mathbb{R}^{d}} \mathbf{1}\left\{\forall i: \boldsymbol{x}_{i} \in 2(1+\beta) B_{a}\right\} \mathbf{1}\left\{Z\left(\boldsymbol{x}_{1}, \ldots, \boldsymbol{x}_{N}\right) \subset(1+\beta) B_{a}\right\} \\
& \quad \times \mathbf{1}\left\{v_{k}\left(Z\left(\boldsymbol{x}_{1}, \ldots, \boldsymbol{x}_{N}\right)\right) \in a(1,1+h)\right\} \mathrm{d} \boldsymbol{x}_{1} \ldots \mathrm{d} \boldsymbol{x}_{N} .
\end{aligned}
$$

Assume that $\boldsymbol{x}_{1}, \ldots, \boldsymbol{x}_{N} \in \mathbb{R}^{d}$ satisfy the conditions

(i) $\left(\boldsymbol{x}_{1}, \ldots, \boldsymbol{x}_{N}\right) \in\left\|\boldsymbol{x}_{N}\right\| \mathcal{Q}_{N}$;

(ii) $v_{k}\left(Z\left(\boldsymbol{x}_{1}, \ldots, \boldsymbol{x}_{N}\right)\right) \in a(1,1+h)$.

Then, using (ii), (i) and the definition of $\mathcal{Q}_{N}$, we get

$$
\frac{a(1+h)}{\left\|\boldsymbol{x}_{N}\right\|^{k}} \geq \frac{v_{k}\left(Z\left(\boldsymbol{x}_{1}, \ldots, \boldsymbol{x}_{N}\right)\right)}{\left\|\boldsymbol{x}_{N}\right\|^{k}} \geq 2^{-k} \kappa_{d}
$$

hence

$$
\left\|\boldsymbol{x}_{N}\right\| \leq 2 a^{1 / k} \kappa_{d}^{-1 / k}(1+h)^{1 / k} .
$$

The definition of $h_{0}$ and (15) imply that

$$
\left\|\boldsymbol{x}_{N}\right\| \leq 2(1+\beta) a^{1 / k} \kappa_{d}^{-1 / k} .
$$

Further, using (i), the definition of $\mathcal{Q}_{N},(15)$ and the definition of $h_{0}$, we find that, for $i=1, \ldots, N-1$,

$$
\left\|\boldsymbol{x}_{i}\right\| \leq\left\|\boldsymbol{x}_{N}\right\|(1+\beta / 2) \leq 2 a^{1 / k} \kappa_{d}^{-1 / k}(1+h)^{1 / k}(1+\beta / 2) \leq 2(1+\beta)\left(a / \kappa_{d}\right)^{1 / k},
$$


thus $\boldsymbol{x}_{i} \in 2(1+\beta) B_{a}$. Finally, (i), the definition of $\mathcal{Q}_{N},(15)$ and the definition of $h_{0}$ imply that

$$
\begin{aligned}
Z\left(\boldsymbol{x}_{1}, \ldots, \boldsymbol{x}_{N}\right) & \subset 2^{-1}\left\|\boldsymbol{x}_{N}\right\|(1+\beta / 2) B^{d} \subset\left(a / \kappa_{d}\right)^{1 / k}(1+h)^{1 / k}(1+\beta / 2) B^{d} \\
& \subset(1+\beta) B_{a} .
\end{aligned}
$$

Hence, introducing polar coordinates, we obtain

$$
\begin{aligned}
\mathrm{P} & \left(v_{k}(Z) \in a(1,1+h)\right) \\
\geq & \frac{\lambda^{N}}{N !} \exp \left\{-v_{d}\left(2(1+\beta) B_{a}\right) \lambda\right\} \int_{\mathbb{R}^{d}} \ldots \int_{\mathbb{R}^{d}} \mathbf{1}\left\{\left(\boldsymbol{x}_{1}, \ldots, \boldsymbol{x}_{N}\right) \in\left\|\boldsymbol{x}_{N}\right\| \mathcal{Q}_{N}\right\} \\
& \times \mathbf{1}\left\{v_{k}\left(Z\left(\boldsymbol{x}_{1}, \ldots, \boldsymbol{x}_{N}\right)\right) \in a(1,1+h)\right\} \mathrm{d} \boldsymbol{x}_{1} \ldots \mathrm{d} \boldsymbol{x}_{N} \\
= & \frac{\lambda^{N}}{N !} \exp \left\{-v_{d}\left(2(1+\beta) B_{a}\right) \lambda\right\} \\
& \times \int_{S^{d-1}} \int_{0}^{\infty} \int_{\mathbb{R}^{d}} \ldots \int_{\mathbb{R}^{d}} \mathbf{1}\left\{\left(\boldsymbol{x}_{1}, \ldots, \boldsymbol{x}_{N-1}, r \boldsymbol{u}\right) \in r \mathcal{Q}_{N}\right\} \\
& \times \mathbf{1}\left\{v_{k}\left(Z\left(\boldsymbol{x}_{1}, \ldots, \boldsymbol{x}_{N-1}, r \boldsymbol{u}\right)\right) \in a(1,1+h)\right\} r^{d-1} \mathrm{~d} \boldsymbol{x}_{1} \ldots \mathrm{d} \boldsymbol{x}_{N-1} \mathrm{~d} r \sigma(\mathrm{d} \boldsymbol{u}) .
\end{aligned}
$$

Substituting $\boldsymbol{x}_{i}=r \boldsymbol{y}_{i}$ for $i=1, \ldots, N-1$, we get

$$
\begin{aligned}
\mathrm{P} & \left(v_{k}(Z) \in a(1,1+h)\right) \\
\geq & \frac{\lambda^{N}}{N !} \exp \left\{-v_{d}\left(2(1+\beta) B_{a}\right) \lambda\right\} \\
& \times \int_{S^{d-1}} \int_{0}^{\infty} \int_{\mathbb{R}^{d}} \ldots \int_{\mathbb{R}^{d}} \mathbf{1}\left\{\left(\boldsymbol{y}_{1}, \ldots, \boldsymbol{y}_{N-1}, \boldsymbol{u}\right) \in \mathcal{Q}_{N}\right\} \\
& \times \mathbf{1}\left\{r^{k} v_{k}\left(Z\left(\boldsymbol{y}_{1}, \ldots, \boldsymbol{y}_{N-1}, \boldsymbol{u}\right)\right) \in a(1,1+h)\right\} r^{N d-1} \mathrm{~d} \boldsymbol{y}_{1} \ldots \mathrm{d} \boldsymbol{y}_{N-1} \mathrm{~d} r \sigma(\mathrm{d} \boldsymbol{u}) \\
= & \frac{\lambda^{N}}{N !} \exp \left\{-v_{d}\left(2(1+\beta) B_{a}\right) \lambda\right\} \\
& \times \int_{S^{d-1}} \int_{\mathbb{R}^{d}} \ldots \int_{\mathbb{R}^{d}} \mathbf{1}\left\{\left(\boldsymbol{y}_{1}, \ldots, \boldsymbol{y}_{N-1}, \boldsymbol{u}\right) \in \mathcal{Q}_{N}\right\} \\
& \times \frac{a^{N d / k}}{N d} v_{k}\left(Z\left(\boldsymbol{y}_{1}, \ldots, \boldsymbol{y}_{N-1}, \boldsymbol{u}\right)\right)^{-N d / k}\left((1+h)^{N d / k}-1\right) \mathrm{d} \boldsymbol{y}_{1} \ldots \mathrm{d} \boldsymbol{y}_{N-1} \sigma(\mathrm{d} \boldsymbol{u}) \\
\geq & \frac{\lambda^{N}}{N !} \exp \left\{-v_{d}\left(2(1+\beta) B_{a}\right) \lambda\right\} \frac{a^{N d / k}}{N d}\left(2^{k}(1+\beta / 2)^{-k} \kappa_{d}^{-1}\right)^{N d / k} \frac{N d}{k} h c_{12}(d, \beta) \\
\geq & h\left(a^{d / k} \lambda\right)^{N} \exp \left\{-v_{d}\left(2(1+\beta) B_{a}\right) \lambda\right\} c_{13}(d, \beta),
\end{aligned}
$$

where also (14) was used. Since $v_{d}\left(2(1+\beta) B_{a}\right)=(1+\beta)^{d} 2^{d} \kappa_{d}^{1-d / k} a^{d / k}$, this proves the assertion. 


\section{Probabilities involving elongated cells}

We will later need estimates showing that typical cells which, compared to their value of $v_{k}$, are 'too long', occur only with small probability. This requires the following preparations.

We denote by $\mathcal{P}_{o}^{d} \subset \mathcal{K}_{o}^{d}$ the subset of convex polytopes and by $G(d, k)$ the Grassmannian of $k$-dimensional linear subspaces of $\mathbb{R}^{d}$. For $K \in \mathcal{K}_{o}^{d}$ and $L \in G(d, k)$, the set $K \mid L$ is the image of $K$ under orthogonal projection to $L$. For $k \in\{1, \ldots, d\}$, we define

$$
\eta_{k}(K):=\min \{D(K) / \Delta(K, L): L \in G(d, k)\},
$$

where $D(K)$ denotes the diameter of $K$ and $\Delta(K, L)$ is the width of $K \mid L$ evaluated in $L$.

Let $a>0$ be given. For $m \in \mathbb{N}$, we set

$$
\mathcal{K}_{a}^{d, k}(m):=\left\{K \in \mathcal{K}_{o}^{d}: v_{k}(K) \in a(1,2), \eta_{k}(K) \in\left[m^{k},(m+1)^{k}\right)\right\} .
$$

Lemma 3. Let $m \in \mathbb{N}$ and $k \in\{1, \ldots, d\}$. Then

(a) $K \in \mathcal{K}_{a}^{d, k}(m)$ implies that $K \subset c_{14} m^{k} a^{1 / k} B^{d}=: C$;

(b) there exists a measurable map $\mathcal{K}_{a}^{d, k}(m) \cap \mathcal{P}_{o}^{d} \ni P \mapsto \boldsymbol{v}(P)$ such that $\boldsymbol{v}(P)$ is a vertex of $P$ with $\|\boldsymbol{v}(P)\| \geq c_{15} m a^{1 / k}$.

Proof. We use repeatedly that $v_{k}(K \mid L)$ is a constant multiple of the $k$-dimensional volume of $K \mid L$.

(a) A special case of equation (5.3.23) in [15] and the monotoneity of mixed volumes imply that

$$
v_{k}(K \mid L) \leq c_{16} v_{k}(K)
$$

holds for all $K \in \mathcal{K}_{o}^{d}$ and $L \in G(d, k)$. Let $K \in \mathcal{K}_{a}^{d, k}(m)$, and choose $L_{0} \in G(d, k)$ such that $\eta_{k}(K)=D(K) / \Delta\left(K, L_{0}\right)$. Then

$$
2 a \geq v_{k}(K) \geq c_{16}^{-1} v_{k}\left(K \mid L_{0}\right) \geq c_{17} \Delta\left(K, L_{0}\right)^{k-1} D\left(K \mid L_{0}\right),
$$

where the estimate (16) from [7] was used. Thus

$$
2 a \geq c_{17} \Delta\left(K, L_{0}\right)^{k} \geq c_{17}(m+1)^{-k^{2}} D(K)^{k}
$$

and therefore

$$
D(K) \leq c_{18} m^{k} a^{1 / k}
$$

Since $\boldsymbol{o} \in K$, this implies (a).

(b) For any $L \in G(d, k)$, we enclose $K \mid L$ in a rectangular parallelepiped in $L$ with one edge length equal to $\Delta(K, L)$ and the other edge lengths at most $D(K \mid L)$. Then

$$
v_{k}(K \mid L) \leq c_{19} \Delta(K, L) D(K \mid L)^{k-1} \leq c_{19} m^{-k} D(K) D(K)^{k-1}
$$

and hence, by an integral-geometric projection formula ([15], (5.3.27)),

$$
a \leq v_{k}(K) \leq c_{20} m^{-k} D(K)^{k}
$$


Therefore, $K$ has a point at distance at least $2^{-1} c_{20}^{-1 / k} m a^{1 / k}$ from the origin. If $K$ is a polytope, such a point can be chosen as a vertex. That a measurable selection is possible, follows as in [7, Lemma 3.5(c)].

Remark. We have $\eta_{1}(K)=1$; moreover, $\mathcal{K}_{a}^{d, 1}(m) \neq \emptyset$ only for $m=1$. Therefore, some of the subsequent arguments simplify considerably, or can be omitted, in the case $k=1$.

Let $a>0, \epsilon>0$ be given. For $m \in \mathbb{N}$, we define

$$
\mathcal{K}_{a, \epsilon}^{d, k}(m):=\left\{K \in \mathcal{K}_{a}^{d, k}(m): \delta(K) \geq \epsilon\right\}
$$

and

$$
q_{a, \epsilon}^{k}(m):=\mathrm{P}\left(Z \in \mathcal{K}_{a, \epsilon}^{d, k}(m)\right) .
$$

Similarly as in [7], we prove two estimates concerning the decay of $q_{a, \epsilon}^{k}(m)$ as $a^{d / k} \lambda \rightarrow \infty$. The dependence on $\epsilon$ will not play a role until Lemma 6 .

For $\boldsymbol{u}_{1}, \ldots, \boldsymbol{u}_{n} \in S^{d-1}$ and $t_{1}, \ldots, t_{n} \in(0, \infty)$ we introduce the abbreviation

$$
\bigcap_{i=1}^{n} H^{-}\left(\boldsymbol{u}_{i}, t_{i}\right)=: P\left(\boldsymbol{u}_{(n)}, t_{(n)}\right) \text {. }
$$

Lemma 4. For $m \in \mathbb{N}$ and $a^{d / k} \lambda \geq \sigma_{0}>0$,

$$
q_{a, \epsilon}^{k}(m) \leq c_{21}\left(d, \sigma_{0}\right) \exp \left\{-c_{22} m^{d} a^{d / k} \lambda\right\}
$$

Proof. Let $C$ be the ball defined in Lemma 3(a). Then

$$
q_{a, \epsilon}^{k}(m)=\sum_{N=d+1}^{\infty} \mathrm{P}\left(Y\left(\mathcal{H}_{C}\right)=N\right) \mathrm{P}\left(Z \in \mathcal{K}_{a, \epsilon}^{d, k}(m) \mid Y\left(\mathcal{H}_{C}\right)=N\right) .
$$

Here,

$$
\begin{aligned}
p_{N}:= & \mathrm{P}\left(Z \in \mathcal{K}_{a, \epsilon}^{d, k}(m) \mid Y\left(\mathcal{H}_{C}\right)=N\right) \\
= & \frac{1}{U(C)^{N}} \int_{S^{d-1}} \int_{0}^{\infty} \cdots \int_{S^{d-1}} \int_{0}^{\infty} \mathbf{1}\left\{P\left(\boldsymbol{u}_{(N)}, t_{(N)}\right) \in \mathcal{K}_{a, \epsilon}^{d, k}(m)\right\} \\
& \times \mathbf{1}\left\{\forall i: H\left(\boldsymbol{u}_{i}, t_{i}\right) \cap C \neq \emptyset\right\}\left(t_{1} \cdots t_{N}\right)^{d-1} \mathrm{~d} t_{1} \sigma\left(\mathrm{d} \boldsymbol{u}_{1}\right) \ldots \mathrm{d} t_{N} \sigma\left(\mathrm{d} \boldsymbol{u}_{N}\right) .
\end{aligned}
$$

Suppose that $\boldsymbol{u}_{1}, \ldots, \boldsymbol{u}_{N}, t_{1}, \ldots, t_{N}$ are such that the indicator functions occurring in the multiple integral are all equal to one; then $P:=P\left(\boldsymbol{u}_{(N)}, t_{(N)}\right) \in \mathcal{K}_{a, \epsilon}^{d, k}(m)$ has a vertex $\boldsymbol{v}(P)$ according to Lemma 3(b). This vertex is the intersection of $d$ facets of $P$. Hence, there exists an index set $J \subset\{1, \ldots, N\}$ with $d$ elements such that

$$
\{\boldsymbol{v}(P)\}=\bigcap_{i \in J} H\left(\boldsymbol{u}_{i}, t_{i}\right) .
$$

The segment $S:=[\boldsymbol{o}, \boldsymbol{v}(P)]$ satisfies

$$
\text { relint } S \cap H\left(\boldsymbol{u}_{j}, t_{j}\right)=\emptyset \quad \text { for } j \in\{1, \ldots, N\} \backslash J .
$$


Since $S \subset C$, we have

$$
\begin{aligned}
& \int_{S^{d-1}} \int_{0}^{\infty} \mathbf{1}\{H(\boldsymbol{u}, t) \cap C \neq \emptyset, H(\boldsymbol{u}, t) \cap S=\emptyset\} t^{d-1} \mathrm{~d} t \sigma(\mathrm{d} \boldsymbol{u}) \\
& =U(C)-U(S)=U(C)-2^{-d} \kappa_{d}|S|^{d}
\end{aligned}
$$

where $|S|$ denotes the length of $S$. Similarly as in the proof of [7, Lemma 3.6] we obtain

$$
\begin{aligned}
p_{N} \leq & \left(\begin{array}{c}
N \\
d
\end{array}\right) \frac{1}{U(C)^{N}} \int_{S^{d-1}} \int_{0}^{\infty} \cdots \int_{S^{d-1}} \int_{0}^{\infty} \mathbf{1}\left\{H\left(\boldsymbol{u}_{i}, t_{i}\right) \cap C \neq \emptyset, i=1, \ldots, d\right\} \\
& \times\left[U(C)-c_{23} m^{d} a^{d / k}\right]^{N-d}\left(t_{1} \cdots t_{d}\right)^{d-1} \mathrm{~d} t_{1} \sigma\left(\mathrm{d} \boldsymbol{u}_{1}\right) \ldots \mathrm{d} t_{d} \sigma\left(\mathrm{d} \boldsymbol{u}_{d}\right) \\
= & \left(\begin{array}{l}
N \\
d
\end{array}\right)\left(1-\frac{c_{23} m^{d} a^{d / k}}{U(C)}\right)^{N-d} .
\end{aligned}
$$

This leads to the estimate

$$
\begin{aligned}
q_{a, \epsilon}^{k}(m) \leq & \sum_{N=d+1}^{\infty} \frac{\left[2^{d} U(C) \lambda\right]^{N}}{N !} \exp \left\{-2^{d} U(C) \lambda\right\}\left(\begin{array}{c}
N \\
d
\end{array}\right)\left(1-\frac{c_{23} m^{d} a^{d / k}}{U(C)}\right)^{N-d} \\
= & \frac{1}{d !}\left[2^{d} U(C) \lambda\right]^{d} \exp \left\{-2^{d} U(C) \lambda\right\} \\
& \times \sum_{N=d+1}^{\infty} \frac{1}{(N-d) !}\left[2^{d} U(C) \lambda-c_{24} m^{d} a^{d / k} \lambda\right]^{N-d} \\
\leq & \frac{1}{d !}\left[2^{d} U(C) \lambda\right]^{d} \exp \left\{-c_{24} m^{d} a^{d / k} \lambda\right\} \\
\leq & c_{25} m^{k d^{2}}\left(a^{d / k} \lambda\right)^{d} \exp \left\{-c_{24} m^{d} a^{d / k} \lambda\right\} \\
\leq & c_{27}\left(d, \sigma_{0}\right) \exp \left\{-c_{26} m^{d} a^{d / k} \lambda\right\}
\end{aligned}
$$

which completes the proof.

For a polytope $P$, let $\operatorname{ext} P$ be the set of vertices and $f_{0}(P)$ the number of vertices of $P$.

Lemma 5. Let $\alpha>0$ be given. There is a number $\nu \in \mathbb{N}$ depending only on $d$ and $\alpha$ such that the following is true. For $P \in \mathcal{P}_{o}^{d}$ there exists a polytope $L=L(P) \in \mathcal{P}_{o}^{d}$ satisfying $\operatorname{ext} L \subset \operatorname{ext} P, f_{0}(L) \leq \nu$, and $U(L) \geq(1-\alpha) U(P)$. Moreover, there exists a measurable selection $P \mapsto L(P)$.

Proof. The following can be extracted from the proof of Lemma 4.2 in [7]. There exist numbers $k_{0}=k_{0}(d)$ and $b_{0}=b_{0}(d)$ such that the following is true. Let $P \in \mathcal{P}_{o}^{d}$ be a polytope and let $P \subset R B^{d}$, where $R$ is minimal. Let $k \geq k_{0}$. There is a measurable map $P \mapsto L(P)$ such that $L=L(P)$ is the convex hull of at most $(k+1) d$ vertices of $P, \boldsymbol{o} \in L$, and $P \subset L+\kappa R B^{d}$ with $\kappa=b_{0} k^{-2 /(d-1)}$. 
There is a unit vector $\boldsymbol{u}$ such that $R[\boldsymbol{o}, \boldsymbol{u}] \subset P$ and hence $U(P) \geq U(R[\boldsymbol{o}, \boldsymbol{u}])=2^{-d} \kappa_{d} R^{d}$. By a suitable choice of $k$, depending only on $d$ and $\alpha$, we can achieve that $\kappa \leq 1$ and $4^{d} \kappa \leq \alpha$. Since $h_{L} \leq R$, we get

$$
U(P) \leq \frac{1}{d} \int_{S^{d-1}}\left[h_{L}+\kappa R\right]^{d} \mathrm{~d} \sigma \leq U(L)+\kappa \cdot 2^{d} \kappa_{d} R^{d} \leq U(L)+4^{d} \kappa U(P),
$$

thus $U(L) \geq(1-\alpha) U(P)$, and $\nu=(k+1) d$ is the required number.

Lemma 6. For $m \in \mathbb{N}, \epsilon \in(0,1)$ and $a, \lambda>0$,

$$
q_{a, \epsilon}^{k}(m) \leq c_{28}(d, \epsilon) m^{k d^{2} \nu} \exp \left\{-\left(1+c_{29} \epsilon^{(d+3) / 2}\right) 2^{d} \kappa_{d}^{1-d / k} a^{d / k} \lambda\right\}
$$

where $\nu$ depends only on $d$ and $\epsilon$.

Proof. We define $C$ as in Lemma 3(a) and use (19) and (20) again. Assume that $\boldsymbol{u}_{1}, \ldots, \boldsymbol{u}_{N}, t_{1}, \ldots, t_{N}$ are such that the indicator functions in (20) are all equal to one. Then, by Lemma 1 ,

$$
U\left(P\left(\boldsymbol{u}_{(N)}, t_{(N)}\right)\right) \geq\left(1+\gamma \epsilon^{(d+3) / 2}\right) \kappa_{d}^{1-d / k} a^{d / k} .
$$

Let $\alpha:=\gamma \epsilon^{(d+3) / 2} /\left(2+\gamma \epsilon^{(d+3) / 2}\right)$; then $(1-\alpha)\left(1+\gamma \epsilon^{(d+3) / 2}\right)=1+\alpha$. Put $c_{30}:=\gamma /(2+\gamma)$; then $\alpha>c_{30} \epsilon^{(d+3) / 2}$. By Lemma 5 , there are $\nu=\nu(d, \epsilon)$ vertices of $P\left(\boldsymbol{u}_{(N)}, t_{(N)}\right)$ such that the convex hull $L=L\left(P\left(\boldsymbol{u}_{(N)}, t_{(N)}\right)\right.$ of these vertices satisfies

$$
U(L) \geq(1-\alpha) U\left(P\left(\boldsymbol{u}_{(N)}, t_{(N)}\right)\right) .
$$

The inequalities (23) and (24) imply that

$$
U(L) \geq(1+\alpha) \kappa_{d}^{1-d / k} a^{d / k} .
$$

Now the same argument as in the proof of Lemma 5.2 in [7], with the obvious modifications, yields

$$
\begin{aligned}
& \mathrm{P}\left(Z \in \mathcal{K}_{a, \epsilon}^{d, k}(m) \mid Y\left(\mathcal{H}_{C}\right)=N\right)[U(C)]^{N} \\
& \leq \sum_{j=d+1}^{d \nu}\left(\begin{array}{c}
N \\
j
\end{array}\right)\left(\begin{array}{l}
j \\
d
\end{array}\right)^{\nu}\left[U(C)-(1+\alpha) \kappa_{d}^{1-d / k} a^{d / k}\right]^{N-j}[U(C)]^{j}
\end{aligned}
$$

Here $j$ denotes the number of hyperplanes generating the vertices of $L$, and $\left(\begin{array}{l}j \\ d\end{array}\right)$ is the number of points of intersection of these hyperplanes; thus $\left(\begin{array}{l}j \\ d\end{array}\right)^{\nu}$ estimates the possibilities to choose the vertices of $L$. The probability that the other $N-j$ hyperplanes intersecting $C$ do not meet $L$ is given by $[U(C)-U(L)]^{N-j} U(C)^{-N+j}$, which is estimated using (25).

Inserting the inequality in (19), we can continue as in [7], finally using $U(C)=$ $\left(c_{14} m^{k} a^{1 / k}\right)^{d} \kappa_{d}$ and $\alpha>c_{30} \epsilon^{(d+3) / 2}$. This completes the proof.

\section{Proof of Theorem 1}

From now on, the proofs follow essentially the lines of those given in [7]. We will, therefore, state only the necessary lemmas in their modified forms and refer to the corresponding proofs in [7]. 
Let $a>0$ and $\epsilon \in(0,1)$ be given. For $h \in(0,1]$ and $m \in \mathbb{N}$ we define

$$
\mathcal{K}_{a, \epsilon, h}^{d, k}(m):=\left\{K \in \mathcal{K}_{o}^{d}: v_{k}(K) \in a(1,1+h), \eta_{k}(K) \in\left[m^{k},(m+1)^{k}\right), \delta(K) \geq \epsilon\right\} ;
$$

thus

$$
\mathrm{P}\left(v_{k}(Z) \in a(1,1+h), \delta(K) \geq \epsilon\right)=\sum_{m=1}^{\infty} q_{a, \epsilon}^{k, h}(m)
$$

with

$$
q_{a, \epsilon}^{k, h}(m):=\mathrm{P}\left(Z \in \mathcal{K}_{a, \epsilon, h}^{d, k}(m)\right)
$$

Moreover, we put

$$
q_{a, \epsilon}^{k, h}(m, n):=\mathrm{P}\left(Z \in \mathcal{K}_{a, \epsilon, h}^{d, k}(m), f_{d-1}(Z)=n\right)
$$

for $n \in \mathbb{N}$; here $f_{d-1}(P)$ denotes the number of facets of a polytope $P$. Then we have

$$
\mathrm{P}\left(v_{k}(Z) \in a(1,1+h), \delta(K) \geq \epsilon\right)=\sum_{m=1}^{\infty} \sum_{n=d+1}^{\infty} q_{a, \epsilon}^{k, h}(m, n) .
$$

Finally, we define

$$
\begin{aligned}
R_{a, \epsilon}^{k, h}(m, n):= & \left\{\left(\boldsymbol{u}_{1}, \ldots, \boldsymbol{u}_{n}, t_{1}, \ldots, t_{n}\right) \in\left(S^{d-1}\right)^{n} \times(0, \infty)^{n}:\right. \\
& P\left(\boldsymbol{u}_{(n)}, t_{(n)}\right) \in \mathcal{K}_{a, \epsilon, h}^{d, k}(m), f_{d-1}\left(P\left(\boldsymbol{u}_{(n)}, t_{(n)}\right)\right)=n, \\
& \left.H\left(\boldsymbol{u}_{i}, t_{i}\right) \cap C \neq \emptyset \quad \text { for } i=1, \ldots, n\right\},
\end{aligned}
$$

where the ball $C$ is again defined as in Lemma 3(a), for the given $a, \epsilon, m$.

Lemma 7. For $m, n \in \mathbb{N}, n \geq d+1$ and $h \in(0,1]$,

$$
\begin{aligned}
q_{a, \epsilon}^{k, h}(m, n)= & \frac{\left(2^{d} \lambda\right)^{n}}{n !} \underbrace{\int \cdots \int}_{R_{a, \epsilon}^{k, h}(m, n)} \exp \left\{-2^{d} U\left(P\left(\boldsymbol{u}_{(n)}, t_{(n)}\right)\right) \lambda\right\} \\
& \times\left(t_{1} \cdots t_{n}\right)^{d-1} \mathrm{~d} t_{1} \ldots \mathrm{d} t_{n} \sigma\left(\mathrm{d} \boldsymbol{u}_{1}\right) \ldots \sigma\left(\mathrm{d} \boldsymbol{u}_{n}\right) .
\end{aligned}
$$

The proof is the same as that for Lemma 6.1 in [7], with the obvious necessary changes.

Lemma 8. Let $w>0, h \in(0,1 / 2)$ and $r \geq d-1$. Then

$$
\begin{aligned}
& \int_{1}^{\sqrt[k]{1+h}} x^{r} \exp \left\{-w x^{d}\right\} \mathrm{d} x \\
& \leq c_{20} h w\left[1+(\exp \{w / 2\}-1)^{-1}\right] \int_{1}^{\sqrt[k]{2}} x^{r} \exp \left\{-w x^{d}\right\} \mathrm{d} x .
\end{aligned}
$$

After the substitution $x^{d}=y$, one can imitate the proof of Lemma 6.2 in [7] to obtain the result. 
The next (technical) lemma states that each bound for $q_{a, \epsilon}^{k, 1}$ yields a bound for $q_{a, \epsilon}^{k, h}$ which is linear in $h$. This should be compared to the bound in Lemma 2 which is also linear in $h$.

Lemma 9. For $m \in \mathbb{N}, h \in(0,1 / 2)$ and $a^{d / k} \lambda \geq \sigma_{0}>0$,

$$
q_{a, \epsilon}^{k, h}(m) \leq c_{31}\left(d, \sigma_{0}\right) h a^{d / k} \lambda m^{d k} q_{a, \epsilon}^{k, 1}(m) .
$$

Again, the proof is obtained by adapting the corresponding one from [7], namely that of Lemma 6.3. After applying Lemmas 7 and 8, we arrive at the inequality

$$
\begin{aligned}
q_{a, \epsilon}^{k, h}(m, n) \leq & \frac{\left(2^{d} \lambda\right)^{n}}{n !} \int_{U(m, n)} t(\zeta)^{d(n-1)+1} c_{32} h U(K(\zeta, t(\zeta))) \lambda \\
& \times\left(1+\left(\exp \left\{2^{d-1} U(K(\zeta, t(\zeta))) \lambda\right\}-1\right)^{-1}\right) \\
& \times \int_{1}^{\sqrt[k]{2}} s^{d(n-1)} \exp \left\{-2^{d} U(K(\zeta, t(\zeta))) \lambda s^{d}\right\} \mathrm{d} s \\
& \times\left(t_{1} \ldots t_{n-1}\right)^{d-1} \mathrm{~d} t_{1} \ldots \mathrm{d} t_{n-1} \sigma\left(\mathrm{d} \boldsymbol{u}_{1}\right) \ldots \sigma\left(\mathrm{d} \boldsymbol{u}_{n}\right),
\end{aligned}
$$

where $U(m, n), t(\zeta)$ and $K(\cdot, \cdot)$ are defined as in [7], with the obvious changes. Now we have to observe that $v_{k}\left(K(\zeta, t(\zeta))=a\right.$, hence $K(\zeta, t(\zeta)) \in \mathcal{K}_{a, \epsilon}^{d, k}(m)$, which implies that

$$
c_{33} m^{d} a^{d / k} \leq U(K(\zeta, t(\zeta))) \leq c_{34} m^{k d} a^{d / k},
$$

by Lemma 3. The estimation can now be completed as in [7].

The following lemma establishes an upper estimate for an unconditional probability.

Lemma 10. Let $\epsilon \in(0,1), h \in(0,1 / 2)$ and $a^{d / k} \lambda \geq \sigma_{0}>0$. Then

$$
\begin{aligned}
& \mathrm{P}\left(v_{k}(Z) \in a(1,1+h), \delta(Z) \geq \epsilon\right) \\
& \leq c_{35}\left(d, \epsilon, \sigma_{0}\right) h \exp \left\{-\left(1+\left(c_{29} / 2\right) \epsilon^{(d+3) / 2}\right) 2^{d} \kappa_{d}^{1-d / k} a^{d / k} \lambda\right\} .
\end{aligned}
$$

Here $c_{29}$ is the constant appearing in Lemma 6. The proof of Lemma 10 follows the one of Proposition 7.1 in [7] and uses Lemmas 9, 4 and 6, in this order.

The choice $\beta=\left(c_{29} / 4\right) \epsilon^{(d+3) / 2}$ in Lemma 2 immediately proves Theorem 1 with $b=$ $a(1+h)$ in the case $h \leq \min \left(h_{0}, 1 / 2\right)$. As to arbitrary $b \geq a$, we observe that Lemmas 2 and 10 have the same structure as Lemma 3.2 and Proposition 7.1, respectively, in [7]; they differ only by the values of some parameters. It is, therefore, clear that Theorem 1 now follows precisely in the same way as Theorem 1 of [7] was proved.

\section{Proof of Theorem 2}

In this section, $Y$ denotes a stationary isotropic Poisson hyperplane process in $\mathbb{R}^{d}$ with intensity $\lambda>0$. For a convex body $K \subset \mathbb{R}^{d}$ and for $k \in \mathbb{N}$, we have

$$
\mathrm{P}\left(Y\left(\mathcal{H}_{K}\right)=k\right)=\frac{\left[2 \kappa_{d}^{-1} v_{1}(K) \lambda\right]^{k}}{k !} \exp \left\{-2 \kappa_{d}^{-1} v_{1}(K) \lambda\right\},
$$


by $[7,(4)]$, where $B$ is now the ball with surface area 1 , thus $B=\left(d \kappa_{d}\right)^{-1 /(d-1)} B^{d}$. Let $Z_{o}$ be the zero cell of the tessellation induced by $Y$. Let $k \in\{1, \ldots, d\}$ be fixed.

Lemma 11. For each $\beta>0$, there are constants $h_{0}>0, N \in \mathbb{N}$ and $c_{36}>0$, depending only on $\beta$ and $d$, such that for $a>0$ and $0<h<h_{0}$,

$$
\mathrm{P}\left(v_{k}\left(Z_{o}\right) \in a(1,1+h)\right) \geq c_{36} h\left(a^{1 / k} \lambda\right)^{N} \exp \left\{-(1+\beta) 2 \kappa_{d}^{-1 / k} a^{1 / k} \lambda\right\} .
$$

Proof. In order to be able to essentially copy the proof of Lemma 2, we define a measure $\psi$ on $\mathbb{R}^{d}$ by

$$
\psi(A):=\int_{S^{d-1}} \int_{0}^{\infty} \mathbf{1}_{A}(t \boldsymbol{u}) \mathrm{d} t \sigma_{0}(\mathrm{~d} \boldsymbol{u})
$$

for Borel sets $A \subset \mathbb{R}^{d}$; here $\sigma_{0}$ denotes the normalized spherical Lebesgue measure on $S^{d-1}$. Let $\tilde{X}$ be the Poisson process in $\mathbb{R}^{d}$ with intensity measure $\lambda \psi$, and let $Y^{\prime}$ be the hyperplane process defined by $Y^{\prime}:=\{H(\boldsymbol{x}): \boldsymbol{x} \in \tilde{X}\}$. Then $Y^{\prime}$ is a Poisson process in the space $\mathcal{H}$ of hyperplanes, and for a Borel set $\mathcal{A} \subset \mathcal{H}$ we have

$$
\begin{aligned}
\mathrm{E} Y^{\prime}(\mathcal{A}) & =\lambda \psi\left(\left\{\boldsymbol{x} \in \mathbb{R}^{d}: H(\boldsymbol{x}) \in \mathcal{A}\right\}\right) \\
& =2 \lambda \int_{S^{d-1}} \int_{0}^{\infty} \mathbf{1}_{\mathcal{A}}(H(\boldsymbol{u}, t)) \mathrm{d} t \sigma_{0}(\mathrm{~d} \boldsymbol{u}) .
\end{aligned}
$$

This shows that $Y^{\prime}$ has the same intensity measure as $Y$. Since $Y$ and $Y^{\prime}$ are Poisson processes, they are stochastically equivalent. We can now repeat the proof of Lemma 2, where we replace $Z$ by $Z_{o}$ and $\mathrm{d} \boldsymbol{x}$ by $\psi(\mathrm{d} \boldsymbol{x})$. Further, we observe that $\psi(\mathrm{d}(r \boldsymbol{u}))=\left(d \kappa_{d}\right)^{-1} \mathrm{~d} r \sigma(\mathrm{d} \boldsymbol{u})$ for $\|\boldsymbol{u}\|=1$ and $\psi(\mathrm{d}(r \boldsymbol{y}))=r \psi(\mathrm{d} \boldsymbol{y})$ for $r>0$. In the exponential, $v_{d}\left(2(1+\beta) B_{a}\right)$ (where $\left.B_{a}=\left(a / \kappa_{d}\right)^{1 / k} B^{d}\right)$ has to be replaced by $\psi\left(2(1+\beta) B_{a}\right)=2(1+\beta)\left(a / \kappa_{d}\right)^{1 / k}$. With these changes, the proof of Lemma 2 yields the assertion of Lemma 11.

We will need a stability version of the inequality (5).

Lemma 12. There is a positive constant $\gamma$, depending only on the dimension $d$, such that for $\epsilon \in(0,1)$ and every convex body $K \subset \mathbb{R}^{d}$ with $r_{d}(K) \geq \epsilon$, the inequality

$$
v_{1}(K) \geq\left(1+\gamma \epsilon^{(d+3) / 2}\right) \kappa_{d}^{1-1 / k} v_{k}(K)^{1 / k}
$$

holds for $k=2, \ldots, d$.

Proof. Without loss of generality, we assume that $K$ has interior points, mean width 2 (the same as the unit ball), and Steiner point $\boldsymbol{o}$. We put $v_{i}:=v_{i}(K)$ for $i=0, \ldots, d\left(v_{0}=\kappa_{d}\right)$ and use the Aleksandrov-Fenchel inequalities $v_{i}^{2} \geq v_{i-1} v_{i+1}$ for $i=1, \ldots, d-1$ (see [15]). Theorem 6.6.6 and Lemma 6.6.5 of [15] provide an improvement of the first of these inequalities, namely

$$
v_{1}^{2}-v_{0} v_{2} \geq c_{37} \delta\left(K, B^{d}\right)^{(d+3) / 2},
$$

where $\delta$ is the Hausdorff distance. This can be rewritten as

$$
\frac{v_{1}}{v_{0}} \geq \frac{v_{2}}{v_{1}}\left(1+\frac{c_{37}}{v_{0} v_{2}} \delta\left(K, B^{d}\right)^{(d+3) / 2}\right) \geq \frac{v_{2}}{v_{1}}(1+\alpha)
$$


with $\alpha:=c_{38} \delta\left(K, B^{d}\right)^{(d+3) / 2}$, since $v_{0} v_{2} \leq v_{1}^{2}=c_{39}\left(v_{1}\right.$ being a constant multiple of the mean width). From

$$
\frac{v_{1}}{v_{0}} \geq(1+\alpha) \frac{v_{2}}{v_{1}} \geq \cdots \geq(1+\alpha) \frac{v_{k}}{v_{k-1}}
$$

we get

$$
\left(\frac{v_{1}}{v_{0}}\right)^{k-1} \geq(1+\alpha)^{k-1} \frac{v_{k}}{v_{1}}
$$

and thus

$$
\frac{v_{1}^{k}}{v_{0}^{k-1} v_{k}} \geq 1+c_{38} \delta\left(K, B^{d}\right)^{(d+3) / 2}
$$

If $\delta:=\delta\left(K, B^{d}\right) \geq 1 / 2$, then (since $\epsilon<1$ )

$$
\frac{v_{1}^{k}}{v_{0}^{k-1} v_{k}} \geq 1+c_{40} \epsilon^{(d+3) / 2} .
$$

If $\delta \leq 1 / 2$, let $r B^{d} \subset K \subset s B^{d}$ where $r$ is maximal and $s$ is minimal. Then (by the definition of the Hausdorff metric) $s \leq 1+\delta$ and $r \geq 1-\delta \geq 1 / 2$, hence $\epsilon \leq r_{d}(K) \leq(s / r)-1 \leq 4 \delta$ and thus

$$
\frac{v_{1}^{k}}{v_{0}^{k-1} v_{k}} \geq 1+c_{41} \epsilon^{(d+3) / 2} .
$$

Both cases together give

$$
v_{1} \geq\left(1+c_{42} \epsilon^{(d+3) / 2}\right)^{1 / k} v_{0}^{1-1 / k} v_{k}^{1 / k} \geq\left(1+c_{43} \epsilon^{(d+3) / 2}\right) \kappa_{0}^{1-1 / k} v_{k}^{1 / k} .
$$

Theorem 2 can now be proved in essentially the same way as Theorem 1, and we list only the necessary changes in Sections 5 and 6, in addition to those already mentioned in the proof of Lemma 11. Definitions (16) and (17) are replaced by

$$
\mathcal{K}_{a, \epsilon}^{d, k}(m):=\left\{K \in \mathcal{K}_{a}^{d, k}(m): r_{d}(K) \geq \epsilon\right\}, \quad q_{a, \epsilon}^{k}(m):=\mathrm{P}\left(Z_{o} \in \mathcal{K}_{a, \epsilon}^{d, k}(m)\right) .
$$

Lemma 13. For $m \in \mathbb{N}$ and $a^{1 / k} \lambda \geq \sigma_{0}>0$,

$$
q_{a, \epsilon}^{k}(m) \leq c_{44}\left(d, \sigma_{0}\right) \exp \left\{-c_{45} m a^{1 / k} \lambda\right\} .
$$

Proof. In the proof of Lemma 4, the number $U(C)$ is replaced by $d v_{1}(C)$. In the integrations, $t \mathrm{~d} t$ is replaced by $\mathrm{d} t$. Equation $(21)$ now reads

$$
\begin{aligned}
& \int_{S^{d-1}} \int_{0}^{\infty} \mathbf{1}\{H(\boldsymbol{u}, t) \cap C \neq \emptyset, H(\boldsymbol{u}, t) \cap S=\emptyset\} \mathrm{d} t \sigma(\mathrm{d} \boldsymbol{u}) \\
& =d v_{1}(C)-d v_{1}(S)=d v_{1}(C)-\kappa_{d-1}|S|
\end{aligned}
$$

hence $(22)$ reads

$$
p_{N} \leq\left(\begin{array}{c}
N \\
d
\end{array}\right)\left(1-\frac{c_{46} m a^{1 / k}}{v_{1}(C)}\right)^{N-d}
$$


Continuing as in the proof of Lemma 4, we arrive at (31).

Lemma 14. Let $k \in\{2, \ldots, d\}$. For $m \in \mathbb{N}, \epsilon \in(0,1)$ and $a, \lambda>0$,

$$
q_{a, \epsilon}^{k}(m) \leq c_{47}(d, \epsilon) m^{k d \nu} \exp \left\{-\left(1+c_{48} \epsilon^{(d+3) / 2}\right) 2 \kappa_{d}^{-1 / k} a^{1 / k} \lambda\right\}
$$

where $\nu$ depends only on $d$ and $\epsilon$.

Proof. We use (19) and (20), with the changes already made for the proof of Lemma 13. Assume that $\boldsymbol{u}_{1}, \ldots, \boldsymbol{u}_{N}, t_{1}, \ldots, t_{N}$ are such that the indicator functions in (20) are all equal to one. By Lemma 12,

$$
v_{1}\left(P\left(\boldsymbol{u}_{(N)}, t_{(N)}\right)\right) \geq\left(1+\gamma \epsilon^{(d+3) / 2}\right) \kappa_{d}^{1-1 / k} a^{1 / k} .
$$

We define $\alpha$ as in the proof of Lemma 6 and put $c_{49}:=\gamma /(2+\gamma)$, so that $\alpha>c_{49} \epsilon^{(d+3) / 2}$. By Lemma 4.2 of [7] (with $B=\left(d \kappa_{d}\right)^{-1 /(d-1)} B^{d}$ ), there are $\nu=\nu(d, \epsilon)$ vertices of $P\left(\boldsymbol{u}_{(N)}, t_{(N)}\right)$ such that their convex hull $L=L\left(P\left(\boldsymbol{u}_{(N)}, t_{(N)}\right)\right)$ satisfies

$$
v_{1}(L) \geq(1-\alpha) v_{1}\left(P\left(\boldsymbol{u}_{(N)}, t_{(N)}\right)\right) .
$$

This gives

$$
v_{1}(L) \geq(1+\alpha) \kappa_{d}^{1-1 / k} a^{1 / k} .
$$

As in the proof of Lemma 6 (and of Lemma 5.2 in [7]) we deduce that

$$
\begin{aligned}
& \mathrm{P}\left(Z_{o} \in \mathcal{K}_{a, \epsilon}^{d, k}(m) \mid Y\left(\mathcal{H}_{C}\right)=N\right)\left[d v_{1}(C)\right]^{N} \\
& \leq \sum_{j=d+1}^{d \nu}\left(\begin{array}{c}
N \\
j
\end{array}\right)\left(\begin{array}{l}
j \\
d
\end{array}\right)^{\nu}\left[d v_{1}(C)-d(1+\alpha) \kappa_{d}^{1-1 / k} a^{d / k}\right]^{N-j}\left[d v_{1}(C)\right]^{j}
\end{aligned}
$$

By Lemma 3,

$$
v_{1}(C)=c_{50} m^{k} a^{1 / k} .
$$

The assertion is now obtained as in the proofs quoted above.

The further proof again follows the lines of [7] and of the proof of Theorem 1 . Definitions (26), (27) and (28) are replaced by

$$
\begin{gathered}
\mathcal{K}_{a, \epsilon, h}^{d, k}(m):=\left\{K \in \mathcal{K}_{o}^{d}: v_{k}(K) \in a(1,1+h), \eta_{k}(K) \in\left[m^{k},(m+1)^{k}\right), r_{d}(K) \geq \epsilon\right\}, \\
q_{a, \epsilon}^{k, h}(m):=\mathrm{P}\left(Z_{o} \in \mathcal{K}_{a, \epsilon, h}^{d, k}(m)\right), \\
q_{a, \epsilon}^{k, h}(m, n):=\mathrm{P}\left(Z_{o} \in \mathcal{K}_{a, \epsilon, h}^{d, k}(m), f_{d-1}\left(Z_{o}\right)=n\right)
\end{gathered}
$$

Lemma 7 is replaced by

$$
\begin{aligned}
q_{a, \epsilon}^{k, h}(m, n)= & \frac{(2 \lambda)^{n}}{n !} \underbrace{\int \ldots \int}_{R_{a, \epsilon}^{k, h}(m, n)} \exp \left\{-2 \kappa_{d}^{-1} v_{1}\left(P\left(\boldsymbol{u}_{(n)}, t_{(n)}\right)\right) \lambda\right\} \\
& \times \mathrm{d} t_{1} \ldots \mathrm{d} t_{n} \sigma_{0}\left(\mathrm{~d} \boldsymbol{u}_{1}\right) \ldots \sigma_{0}\left(\mathrm{~d} \boldsymbol{u}_{n}\right) .
\end{aligned}
$$


This is a special case of Lemma 6.1 in [7]. Instead of Lemma 8, we use Lemma 6.2 from [7], with $d$ replaced by $k \geq 2$. Lemma 9 is replaced by the following assertion. For $m \in \mathbb{N}$, $h \in(0,1 / 2)$ and $a^{1 / k} \lambda \geq \sigma_{0}>0$,

$$
q_{a, \epsilon}^{k, h}(m) \leq c_{51}\left(d, \sigma_{0}\right) h a^{1 / k} \lambda m^{k} q_{a, \epsilon}^{k, 1}(m) .
$$

The proof is obtained by adapting the proof of Lemma 6.3 in [7]. In the course of the proof, one has to use that Lemma 3 implies

$$
c_{52} m a^{1 / k} \leq v_{1}(K) \leq c_{53} m^{k} a^{1 / k}
$$

for $K \in \mathcal{K}_{a, \epsilon}^{d, k}(m)$. The counterpart of Lemma 10 now reads as follows. Let $\epsilon \in(0,1)$, $h \in(0,1 / 2)$ and $a^{1 / k} \lambda \geq \sigma_{0}>0$. Then

$$
\begin{aligned}
& \mathrm{P}\left(v_{k}\left(Z_{o}\right) \in a(1,1+h), r_{d}\left(Z_{o}\right) \geq \epsilon\right) \\
& \leq c_{54}\left(d, \epsilon, \sigma_{0}\right) h \exp \left\{-\left(1+\left(c_{48} / 2\right) \epsilon^{(d+3) / 2}\right) 2 \kappa_{d}^{-1 / k} a^{1 / k} \lambda\right\} .
\end{aligned}
$$

With these preliminaries, the proof of Theorem 2 can now be completed in the same way as that of Theorem 1.

\section{Proof of Theorem 3}

In large parts of the proofs of Theorems 1 and 2, only the following properties of the functional $v_{k}$ are used: it is monotone under set inclusion, i.e., $v_{k}\left(K_{1}\right) \leq v_{k}\left(K_{2}\right)$ if $K_{1} \subset K_{2}$, positively homogeneous of degree $k$, i.e., $v_{k}(r K)=r^{k} v_{k}(K)$ for $r \geq 0$, and continuous with respect to the Hausdorff metric. These properties are shared, with $k=1$, by the inradius functionals $\rho_{o}$ and $\rho$. Hence, the corresponding parts of the proofs apply also to Theorem 3. Additional properties of the function $v_{k}$ were only needed for the stability estimates of Lemmas 1 and 12 and for Lemma 3. We replace these lemmas by the following ones.

Lemma 15. There is a positive constant $\gamma$, depending only on the dimension $d$, such that for $\epsilon \in(0,1)$ and every convex body $K \in \mathcal{K}_{o}^{d}$ with $\delta(K) \geq \epsilon$ the inequality

$$
U(K) \geq\left(1+\gamma \epsilon^{(d+1) / 2}\right) \kappa_{d} \rho_{o}(K)^{d}
$$

holds.

Proof. Without loss of generality, we assume that $\rho_{o}(K)=1$. Let $K \subset R_{o} B^{d}$, where $R_{o}$ is minimal. First we assume that $R_{o} \leq 3$. Put $R_{o}=1+h$, then $\epsilon \leq \delta(K) \leq h \leq 2$. Proceeding similarly as in Case 1 of the proof of Lemma 1, we find that

$$
U(K)-U\left(B^{d}\right) \geq c_{55} h^{(d+1) / 2} \geq c_{55} \epsilon^{(d+1) / 2} .
$$

Now suppose that $R_{o}>3$. Then there is a spherical cap $A \subset S^{d-1}$ with $\sigma(A)=c_{56}>0$ on which $h_{K} \geq 2$. It follows that

$$
U(K)-U\left(B^{d}\right) \geq \frac{1}{d} \int_{A}\left(h_{K}^{d}-1\right) \mathrm{d} \sigma \geq c_{57} \epsilon^{(d+1) / 2},
$$


since $\epsilon<1$. Hence, both cases yield

$$
U(K) \geq U\left(B^{d}\right)+c_{58} \epsilon^{(d+1) / 2}=\left(1+c_{59} \epsilon^{(d+1) / 2}\right) \kappa_{d} \rho_{o}(K)^{d} .
$$

Lemma 16. There is a positive constant $\gamma$, depending only on the dimension $d$, such that for $\epsilon \in(0,1)$ and every convex body $K \in \mathcal{K}_{o}^{d}$ with $r_{d}(K) \geq \epsilon$ the inequality

$$
v_{1}(K) \geq\left(1+\gamma \epsilon^{(d+1) / 2}\right) \kappa_{d} \rho(K)
$$

holds.

The proof is similar to that of Lemma 15.

For a replacement of Lemma 3, let $a>0$ be given. For $m \in \mathbb{N}$ we set

$$
\mathcal{K}_{a}^{d}(m):=\left\{K \in \mathcal{K}_{o}: \rho(K) \in a(1,2), D(K) / \rho(K) \in[m, m+1)\right\} .
$$

Lemma 17. Let $m \in \mathbb{N}$. Then

(a) $K \in \mathcal{K}_{a}^{d}(m)$ implies that $K \subset c_{60} m a B^{d}$;

(b) there exists a measurable map $\mathcal{K}_{a}^{d}(m) \cap \mathcal{P}_{o}^{d} \ni P \mapsto \boldsymbol{v}(P)$ such that $\boldsymbol{v}(P)$ is a vertex of $P$ with $\|\boldsymbol{v}(P)\| \geq c_{61}$ ma.

A similar result holds with $\rho_{o}$ instead of $\rho(K)$. The proof is immediate.

With these changes, the proofs of Theorems 1 and 2 yield the proof of Theorem 3 .

\section{References}

[1] Calka, P. (2002) The distributions of the smallest disks containing the Poisson-Voronoi typical cell and the Crofton cell in the plane. Adv. Appl. Prob. 34, 702-717.

[2] Gardner, R.J. and Vassallo, S. (1999) Stability of inequalities in the dual BrunnMinkowski theory. J. Math. Anal. Appl. 231, 568-587.

[3] Goldman, A. (1998) Sur une conjecture de D.G. Kendall concernant la cellule de Crofton du plan et sur sa contrepartie brownienne. Ann. Probab. 26, 1727-1750.

[4] Groemer, H. (1996) Geometric Applications of Fourier Series and Spherical Harmonics. Encyclopedia of Mathematics and Its Applications 61, Cambridge University Press, Cambridge.

[5] Groemer, H. And Schneider, R. (1991) Stability estimates for some geometric inequalities. Bull. London Math. Soc. 23, $67-74$.

[6] Hug, D. And Schneider, R. (2002). Large cells in Poisson-Delaunay tessellations. Discrete Comput. Geom. (to appear.) 
[7] Hug, D., Reitzner, M. And Schneider, R. (2002). The limit shape of the zero cell in a stationary Poisson hyperplane tessellation. Ann. Probab. (to appear).

[8] Kovalenko, I.N. (1997) A proof of a conjecture of David Kendall on the shape of random polygons of large area. (Russian) Kibernet. Sistem. Anal. 1997, 3-10, 187; Engl. transl. Cybernet. Systems Anal. 33, 461-467.

[9] Kovalenko, I.N. (1998) An extension of a conjecture of D.G. Kendall concerning shapes of random polygons to Poisson Voronoï cells. In: Engel, P. et al. (eds.), Voronoï's impact on modern science. Book I. Transl. from the Ukrainian. Kyiv: Institute of Mathematics. Proc. Inst. Math. Natl. Acad. Sci. Ukr., Math. Appl. 212, 266-274.

[10] Kovalenko, I.N. (1999) A simplified proof of a conjecture of D.G. Kendall concerning shapes of random polygons. J. Appl. Math. Stochastic Anal. 12, 301-310.

[11] Miles, R.E. (1995) A heuristic proof of a long-standing conjecture of D.G. Kendall concerning the shapes of certain large random polygons. Adv. Appl. Prob. 27, 397-417.

[12] Møller, J. (1994) Lectures on Random Voronoi Tessellations. Lect. Notes Statist. 87, Springer, New York.

[13] MøLler, J. (1998) A review on probabilistic models and results for Voronoi tessellations. In: Engel, P. et al. (eds.), Voronoï's impact on modern science. Book I. Transl. from the Ukrainian. Kyiv: Institute of Mathematics. Proc. Inst. Math. Natl. Acad. Sci. Ukr., Math. Appl. 212, $254-265$.

[14] Okabe, A., Boots, B., Sugihara, K. and Chiu, S.N. (2000) Spatial Tessellations; Concepts and Applications of Voronoi Diagrams., 2nd ed., Wiley, Chichester.

[15] Schneider, R. (1993) Convex Bodies: the Brunn-Minkowski Theory. Encyclopedia of Mathematics and Its Applications 44, Cambridge University Press, Cambridge.

[16] Schneider, R. And Weil, W. (2000) Stochastische Geometrie. Teubner Skripten zur Mathematischen Stochastik, Teubner, Stuttgart.

[17] Stoyan, D., Kendall, W.S. And Mecke, J. (1995) Stochastic Geometry and its Applications. 2nd ed., Wiley, Chichester. 